\title{
RBF NN-Based Backstepping Adaptive Control for a Class of Nonlinear Systems
}

\author{
Haibo Zhao', Chengguang Wang ${ }^{2}$ \\ ${ }^{1}$ Engineering Technology Research Center of Optoelectronic Appliance, Tongling University, Tongling Anhui, 244061, China \\ ${ }^{2}$ Sichuan Institute of Aerospace System Engineering, Chengdu Sichuan, 610100, China
}

\begin{abstract}
There are many control methods for nonlinear systems, but some of them can not control nonlinear mismatched systems very well. Backstepping control has obvious advantages in controlling nonlinear mismatched systems. Thus we proposed a new radial-basis-function (RBF) neural network-based backstepping adaptive controller combining RBF neural network (RBF NN) and backstepping control for a class of nonlinear mismatched systems. We adopted RBF NN to approximate the system uncertainty. And we analyzed the controller stability using Lyapunov stability theory. Finally we chose sine signal as simulation input signal, simulation results show that the proposed control strategy has better adaptive ability and robustness than PID control, validating the effectivess of the proposed control strategy.
\end{abstract}

\section{Introduction}

Nonlinear systems have been studied by many scholars, and the control methods used are as follows: iterative learing control [1], feedback linearization [2], fuzzy control [3], sliding mode control [4], etc. However, when the system is a nonlinear mismatched system, the disadvantages of the above methods will be revealed.

Backstepping control can be used to control nonlinear mismatched systems [5]. RBF neural network (RBF NN) has universal approximation capability and can approximate arbitrary nonlinear functions with arbitrary precision [6]. The control algorithm combining RBF NN and backstepping control has been successfully applied by scholars [7-11].

In this paper, we use RBF NN-based backstepping control for a class of nonlinear mismatched systems. RBF NN is used to approximate system uncertainty. Simulation results verify the feasibility of the proposed control algorithm.

\section{Problem description}

Consider the following nonlinear mismatched system with strict feedback [12]:

$$
\left\{\begin{array}{l}
\dot{x}_{1}=x_{2}+f_{1}\left(x_{1}\right)+\Delta f_{1}\left(x_{1}\right) \\
\dot{x}_{2}=x_{3}+f_{2}\left(x_{1}, x_{2}\right)+\Delta f_{2}\left(x_{1}, x_{2}\right) \\
\cdots \\
\dot{x}_{n-1}=x_{n}+f_{n-1}\left(x_{1}, x_{2}, \cdots, x_{n-1}\right)+\Delta f_{n-1}\left(x_{1}, x_{2}, \cdots, x_{n-1}\right) \\
\dot{x}_{n}=\psi u+f_{n}\left(x_{1}, x_{2}, \cdots, x_{n}\right)+\Delta f_{n}\left(x_{1}, x_{2}, \cdots, x_{n}\right) \\
y=x_{1}
\end{array}\right.
$$

where $\boldsymbol{x}=\left[x_{1}, x_{2}, \cdots, x_{n}\right]^{\mathrm{T}}$ is a system state variable. $y$ is a system output. $u$ is a system control input. Let $\overline{\boldsymbol{x}}_{i}=\left[x_{1}, x_{2}, \cdots, x_{i}\right]^{\mathrm{T}}$, strict feedback means that the nonlinear terms $f_{i}$ and $\Delta f_{i}$ depend only on the system state $x_{1}, x_{2}, \cdots, x_{i}$.

In addition, the nonlinear terms satisfy the following characteristics : (a) $f_{i}: R^{i} \rightarrow R$ should be smooth enough and $f_{i}(0,0, \cdots, 0)=0, i=1,2, \cdots, n$; (b) $\Delta f_{i}: R^{i} \rightarrow R$ is unknown, $\Delta f_{i} \in C^{l}$ and $\Delta f_{i}(0,0, \cdots, 0)=0$.

Assumption 1 Given a smooth nonlinear function vector $\boldsymbol{h}: \Omega \mapsto R^{n}$ ( $\Omega$ is a compact set in $R^{m}$ ), there exist an optimal Gaussian base function vector $\varphi: R^{m} \mapsto R^{n}$ and weight matrix $\boldsymbol{W}_{n \times n}^{*}$ such that :

$$
\boldsymbol{h}(\boldsymbol{x})=\boldsymbol{W}^{* \mathrm{~T}} \boldsymbol{\varphi}(\boldsymbol{x})+\boldsymbol{\varepsilon}, \forall \boldsymbol{x} \in \Omega
$$

where $\varepsilon$ is the construction error of RBF NN.

In this paper, our main task is to design an adaptive controller so as to make system output $x_{1}$ asymptotically stable tracking the expected output $x_{1 d}$, namely $\lim _{t \rightarrow \infty}\left|x_{1}-x_{1 d}\right|=0$.

\section{Controller design and stability analysis}

We use a RBF NN-based backstepping adaptive control strategy for a class of nonlinear system, Lyapunov stability theory is used to analyze the system stability. The detailed design process is as follows.

Step 1 Define the tracking error between the actual system output $x_{1}$ and the expected system output $x_{1 d}$ as :

$$
e_{1}=x_{1}-x_{1 d}
$$


Its derivative is

$$
\dot{e}_{1}=\dot{x}_{1}-\dot{x}_{1 d}=x_{2}+f_{1}\left(x_{1}\right)+\Delta f_{1}\left(x_{1}\right)-\dot{x}_{1 d}
$$

To eliminate the influence of uncertainty $\Delta f_{1}$, we use RBF NN to approximate $\Delta f_{1}$ :

$$
\Delta f_{1}\left(x_{1}\right)=\boldsymbol{W}_{1}^{* \mathrm{~T}} \boldsymbol{\varphi}_{1}\left(x_{1}\right)+\varepsilon_{1}
$$

where $x_{1}$ is a RBF NN input.

If $x_{2}$ is considered the actual input of system (4), then there exist an ideal control $x_{2}^{*}$ [13]:

$$
x_{2}^{*}=-\left(f_{1}-\dot{x}_{1 d}+k_{1} e_{1}+\boldsymbol{W}_{1}^{* \mathrm{~T}} \boldsymbol{\varphi}_{1}+\varepsilon_{1}\right)
$$

such that $\dot{e}_{1}=-k_{1} e_{1}+\left(x_{2}-x_{2}^{*}\right), \quad k_{1}>0$ is a design parameter. Because $x_{2}^{*}$ is not available, we design a virtual control $x_{2 d}$ as follows.

$$
x_{2 d}=-\left(f_{1}-\dot{x}_{1 d}+k_{1} e_{1}+\hat{\boldsymbol{W}}_{1}^{\mathrm{T}} \boldsymbol{\varphi}_{1}\right)
$$

where $\hat{\boldsymbol{W}}_{1}$ is the estimated value of $\boldsymbol{W}_{1}^{*}$.

We choose the following Lyapunov function $V_{1}$ :

$$
V_{1}=\frac{1}{2} e_{1}^{2}+\frac{1}{2} \operatorname{tr}\left[\tilde{\boldsymbol{W}}_{1}^{\mathrm{T}} \boldsymbol{\Gamma}^{-1} \tilde{\boldsymbol{W}}_{1}\right]
$$

where $\tilde{\boldsymbol{W}}_{1}=\boldsymbol{W}_{1}^{*}-\hat{\boldsymbol{W}}_{1}, \Gamma_{1}=\Gamma_{1}^{\mathrm{T}}>0$ is a design parameter.

The derivative of $V_{1}$ is as follows :

$\dot{V}_{1}=e_{1} \dot{e}_{1}+\operatorname{tr}\left[\tilde{\boldsymbol{W}}_{1}^{T} \Gamma_{1}^{-1} \dot{\tilde{\boldsymbol{W}}}_{1}\right]$

$=-k_{1} e_{1}^{2}+e_{1}\left(x_{2}-x_{2 d}\right)+\tilde{\boldsymbol{W}}_{1}^{\mathrm{T}} \boldsymbol{\varphi}_{1} e_{1}+\varepsilon_{1} e_{1}+\operatorname{tr}\left[\tilde{\boldsymbol{W}}_{1}^{\mathrm{T}} \boldsymbol{\Gamma}_{1}^{-1} \dot{\boldsymbol{W}}_{1}\right]$

Step $i(i=2, \cdots, n-1)$ Define the tracking error $e_{i}$ between the system state $x_{i}$ and the virtual control $x_{i d}$ as

Its derivative is

$$
e_{i}=x_{i}-x_{i d}
$$

$$
\dot{e}_{i}=\dot{x}_{i}-\dot{x}_{i d}=x_{i+1}+f_{i}\left(\bar{x}_{i}\right)+\Delta f_{i}\left(\bar{x}_{i}\right)-\dot{x}_{i d}
$$

Similarly, to eliminate the influence of uncertainty $\Delta f_{i}$, we use RBF NN to approximate $\Delta f_{i}$ :

$$
\Delta f_{i}\left(\bar{x}_{i}\right)=\boldsymbol{W}_{i}^{* \mathrm{~T}} \boldsymbol{\varphi}_{i}\left(\bar{x}_{i}\right)+\varepsilon_{i}
$$

where $\bar{x}_{i}$ is a RBF NN input.

If $x_{i+1}$ is considered the actual input of system (11), then there exist an ideal control $x_{i+1}^{*}$ :

$$
x_{i+1}^{*}=-\left(f_{i}-\dot{x}_{i d}+k_{i} e_{i}+e_{i-1}+\boldsymbol{W}_{i}^{* \mathrm{~T}} \boldsymbol{\varphi}_{i}+\varepsilon_{i}\right)
$$

such that $\dot{e}_{i}=-k_{i} e_{i}-e_{i-1}+\left(x_{i+1}-x_{i+1}^{*}\right), k_{i}>0$ is a design parameter. Because $x_{i+1}^{*}$ is not available, we design a virtual control $x_{i+1, d}$ as follows.

$$
x_{i+1, d}=-\left(f_{i}-\dot{x}_{i d}+k_{i} e_{i}+e_{i-1}+\hat{\boldsymbol{W}}_{i}^{\mathrm{T}} \boldsymbol{\varphi}_{i}\right)
$$

where $\hat{\boldsymbol{W}}_{i}$ is the estimated value of $\boldsymbol{W}_{i}^{*}$.

We choose the following Lyapunov function $V_{i}$ :

$$
V_{i}=\sum_{j=1}^{i-1} V_{j}+\frac{1}{2} e_{i}^{2}+\frac{1}{2} \operatorname{tr}\left[\tilde{\boldsymbol{W}}_{i}^{\mathrm{T}} \boldsymbol{\Gamma}_{i}^{-1} \tilde{\boldsymbol{W}}_{i}\right]
$$

where $\tilde{\boldsymbol{W}}_{i}=\boldsymbol{W}_{i}^{*}-\hat{\boldsymbol{W}}_{i}, \boldsymbol{\Gamma}_{i}=\Gamma_{i}^{\mathrm{T}}>0$ is a design parameter.

The derivative of $V_{i}$ is as follows :

$$
\begin{aligned}
\dot{V}_{i}= & -\sum_{j=1}^{i} k_{j} e_{j}^{2}+e_{i}\left(x_{i+1}-x_{i+1, d}\right) \\
& +\tilde{\boldsymbol{W}}_{i}^{\mathrm{T}} \boldsymbol{\varphi}_{i} e_{i}+\varepsilon_{i} e_{i}+\operatorname{tr}\left[\tilde{\boldsymbol{W}}_{i}^{\mathrm{T}} \boldsymbol{\Gamma}_{i}^{-1} \dot{\tilde{\boldsymbol{W}}}_{i}\right]
\end{aligned}
$$

Step $n$ Define the tracking error $e_{n}$ between the system state $x_{n}$ and the virtual control $x_{n d}$ as

Its derivative is

$$
e_{n}=x_{n}-x_{n d}
$$

$$
\dot{e}_{n}=\dot{x}_{n}-\dot{x}_{n d}=\psi u+f_{n}\left(\bar{x}_{n}\right)+\Delta f_{n}\left(\bar{x}_{n}\right)-\dot{x}_{n d}
$$

To eliminate the influence of uncertainty $\Delta f_{n}$, we use RBF NN to approximate $\Delta f_{n}$ :

$$
\Delta f_{n}\left(\bar{x}_{n}\right)=\boldsymbol{W}_{n}^{* \mathrm{~T}} \boldsymbol{\varphi}_{n}\left(\bar{x}_{n}\right)+\boldsymbol{\varepsilon}_{n}
$$

$u$ is the actual control of system (18), there exist an ideal control $u^{*}$ :

$$
u^{*}=-\left(f_{n}-\dot{x}_{n d}+k_{n} e_{n}+e_{n-1}+\boldsymbol{W}_{n}^{* \mathrm{~T}} \boldsymbol{\varphi}_{n}+\varepsilon_{n}\right)
$$

such that $\dot{e}_{n}=-k_{n} e_{n}-e_{n-1}+\psi\left(u-u^{*}\right), k_{n}>0$ is a design parameter. Because $u^{*}$ is not available, we design an actual control $u$ as follows.

$$
u=-\left(f_{n}-\dot{x}_{n d}+k_{n} e_{n}+e_{n-1}+\hat{\boldsymbol{W}}_{n}^{\mathrm{T}} \boldsymbol{\varphi}_{n}\right)
$$

where $\hat{\boldsymbol{W}}_{n}$ is the estimated value of $\boldsymbol{W}_{n}^{*}$.

We choose the following Lyapunov function $V_{n}$ :

$$
V_{n}=\frac{1}{2} \xi^{\mathrm{T}} \boldsymbol{\xi}+\frac{1}{2} \operatorname{tr}\left[\tilde{\boldsymbol{Z}}^{\mathrm{T}} \boldsymbol{\Gamma}^{-1} \tilde{\boldsymbol{Z}}\right]+\frac{1}{2} \eta \tilde{\psi}^{2}
$$

where $\eta>0$ is a design parameter, $\boldsymbol{\Gamma}=\left[\begin{array}{llll}\boldsymbol{\Gamma}_{1} & & & \\ & \boldsymbol{\Gamma}_{2} & & \\ & & \ddots & \\ & & & \boldsymbol{\Gamma}_{n}\end{array}\right]$, $\boldsymbol{\xi}=\left[e_{1} e_{2} \cdots e_{n}\right]^{\mathrm{T}}$, and $\tilde{\psi}=\psi-\hat{\psi}, \hat{\psi}$ is the estimated value of $\psi$.

We choose the following adaptive law :

$$
\dot{\hat{Z}}=\Gamma \varphi \xi^{\mathrm{T}}-n \boldsymbol{\Gamma}\|\xi\| \hat{\boldsymbol{Z}}
$$

where $\varphi=\left[\varphi_{1} \varphi_{2} \cdots \varphi_{n}\right], n>0$ is a design parameter.

$$
\begin{aligned}
& \boldsymbol{Z}=\left[\begin{array}{llll}
\boldsymbol{W}_{1} & & & \\
& \boldsymbol{W}_{2} & \\
& & \ddots & \\
& & & \boldsymbol{W}_{n}
\end{array}\right],\|\boldsymbol{Z}\|_{F} \leq Z_{M} \\
& \hat{\boldsymbol{Z}}=\left[\begin{array}{llll}
\hat{\boldsymbol{W}}_{1} & & & \\
& \hat{\boldsymbol{W}}_{2} & & \\
& & \ddots & \\
& & & \hat{\boldsymbol{W}}_{n}
\end{array}\right], \hat{\boldsymbol{Z}}=\boldsymbol{Z}-\hat{\boldsymbol{Z}}
\end{aligned}
$$

The derivative of $V_{n}$ is as follows :

$$
\dot{V}_{n}=-\boldsymbol{\xi}^{\mathrm{T}} \boldsymbol{K}_{e} \dot{\boldsymbol{\xi}}+\boldsymbol{\xi}^{\mathrm{T}} \boldsymbol{\varepsilon}+\operatorname{tr}\left[\tilde{\boldsymbol{Z}}^{\mathrm{T}} \boldsymbol{\Gamma}^{-1} \dot{\tilde{\boldsymbol{Z}}}+\tilde{\boldsymbol{Z}}^{\mathrm{T}} \boldsymbol{\varphi} \xi^{\mathrm{T}}\right]+\eta \tilde{\psi} \tilde{\dot{\psi}}
$$
where

$\boldsymbol{K}_{e}=\operatorname{diag}\left\{k_{1}, k_{2}, \cdots, k_{n}\right\}^{\mathrm{T}}, \boldsymbol{\varepsilon}=\left[\varepsilon_{1} \varepsilon_{2} \cdots \varepsilon_{n}\right]^{\mathrm{T}},\|\boldsymbol{\varepsilon}\|<\varepsilon_{N}$.

Since $\dot{\tilde{Z}}=-\dot{\hat{\boldsymbol{Z}}}$, and $\dot{\tilde{\psi}}=-\dot{\hat{\psi}}$, substitute Eq.(23) into (24), we have

$$
\dot{V}_{n}=-\boldsymbol{\xi}^{\mathrm{T}} \boldsymbol{K}_{e} \boldsymbol{\xi}+\boldsymbol{\xi}^{\mathrm{T}} \boldsymbol{\varepsilon}+n\|\boldsymbol{\xi}\| \operatorname{tr}\left[\tilde{\boldsymbol{Z}}^{\mathrm{T}}(\boldsymbol{Z}-\tilde{\boldsymbol{Z}})\right]-\eta \tilde{\psi} \dot{\hat{\psi}}
$$

According to the actual system, the inequality $\tilde{\psi} \dot{\hat{\psi}} \geq 0$ is clearly true. In addition, according to Schwarz inequality, the following inequality is true :

$$
\operatorname{tr}\left[\tilde{\boldsymbol{Z}}^{\mathrm{T}}(\boldsymbol{Z}-\tilde{\boldsymbol{Z}})\right] \leq\|\tilde{\boldsymbol{Z}}\|_{F}\|\boldsymbol{Z}\|_{F}-\|\tilde{\boldsymbol{Z}}\|_{F}^{2}
$$


Since $K_{\min }\|\xi\|^{2} \leq \xi^{T} K_{e} \xi$, where $K_{\min }$ is the minimum eigenvalue of $\boldsymbol{K}_{e}$. Thus the above Eq.(25) becomes :

$$
\begin{aligned}
\dot{V}_{n} & \leq-K_{\text {min }}\|\boldsymbol{\xi}\|^{2}+\varepsilon_{N}\|\xi\|+n\|\boldsymbol{\xi}\|\left(\|\tilde{\boldsymbol{Z}}\|_{F}\|\boldsymbol{Z}\|_{F}-\|\tilde{\boldsymbol{Z}}\|_{F}^{2}\right)-\eta \tilde{\psi} \dot{\hat{\psi}} \\
& \leq-\|\boldsymbol{\xi}\|\left(K_{\text {min }}\|\boldsymbol{\xi}\|-\varepsilon_{N}+n\|\tilde{\boldsymbol{Z}}\|_{F}\left(\|\tilde{\boldsymbol{Z}}\|_{F}-Z_{M}\right)\right)
\end{aligned}
$$

As we know,

$$
\begin{gathered}
K_{\min }\|\xi\|-\varepsilon_{N}+n\left(\|\tilde{\boldsymbol{Z}}\|_{F}^{2}-\|\tilde{\boldsymbol{Z}}\|_{F} Z_{M}\right) \\
=K_{\text {min }}\|\xi\|-\varepsilon_{N}+n\left(\|\tilde{\boldsymbol{Z}}\|_{F}-\frac{1}{2} Z_{M}\right)^{2}-\frac{n}{4} Z_{M}^{2}
\end{gathered}
$$

Thus if the following condition can be met

$$
\|\xi\|>\frac{\varepsilon_{N}+\frac{n}{4} Z_{M}^{2}}{K_{\min }}
$$

then $\dot{V}_{n} \leq 0$.

From Eq.(27), we can see that the tracking accuracy is related to $\varepsilon_{N}, n$ and $K_{\min }$.

\section{Simulation analysis}

We adopt the following system to validate the effectiveness of the proposed control strategy.

$$
\left\{\begin{array}{l}
\dot{x}_{1}=x_{2}+x_{1} \sin \left(x_{1}\right) \\
\dot{x}_{2}=x_{3}+x_{1}^{2}+x_{2}^{2}+\sin \left(x_{1}+x_{2}\right) \\
\dot{x}_{3}=2 u+\sin \left(x_{1}^{2}+x_{2}^{2}+x_{3}^{2}\right) \\
y=x_{1}
\end{array}\right.
$$

The control goal is to make the system output $x_{1}$ trace the desired output $x_{1 d}=0.5 \sin (2 \pi t) . k_{1}=10, k_{2}=8$, $k_{3}=12, \quad \Gamma_{i}(i=1,2,3)$ is a unit array with proper dimension, $\eta=115, n=0.01, \boldsymbol{x}(0)=[0,0,0]^{\mathrm{T}}$. The following curves are comparision curves between PID control and the proposed strategy in this paper, namely RBF NN-based backstepping adaptive control(RBFNNB). Figure 1 and figure 2 are curves of tracking sine signal and error using PID control, respectively. Figure 3 and figure 4 are curves of tracking sine signal and error using RBFNNB, respectively. From figure 1-4, it is obvious for us that RBFNNB has better dynamic and steady performance than PID control.

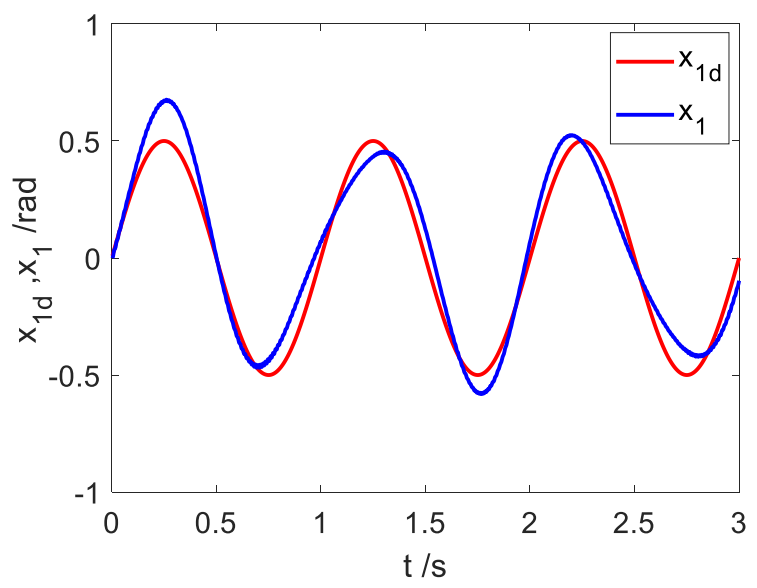

Figure 1. Tracking sine signal using PID control.

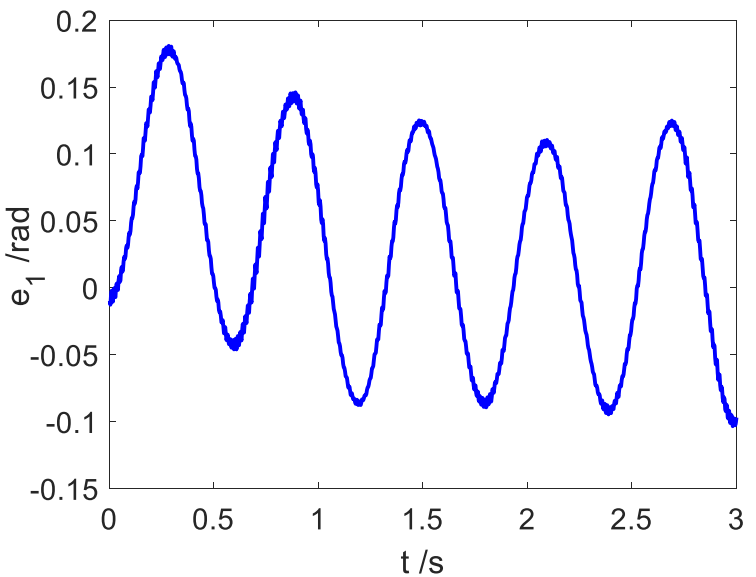

Figure 2. Error of tracking sine signal using PID control.

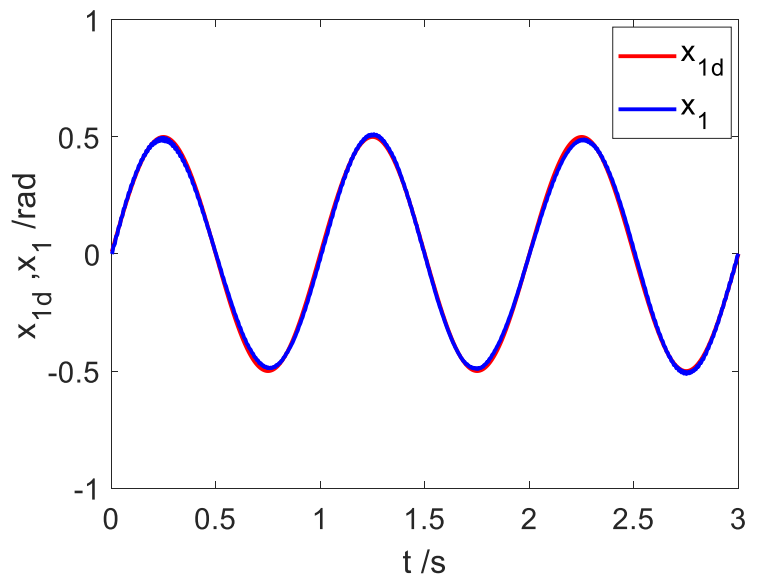

Figure 3. Tracking sine signal using RBFNNB.

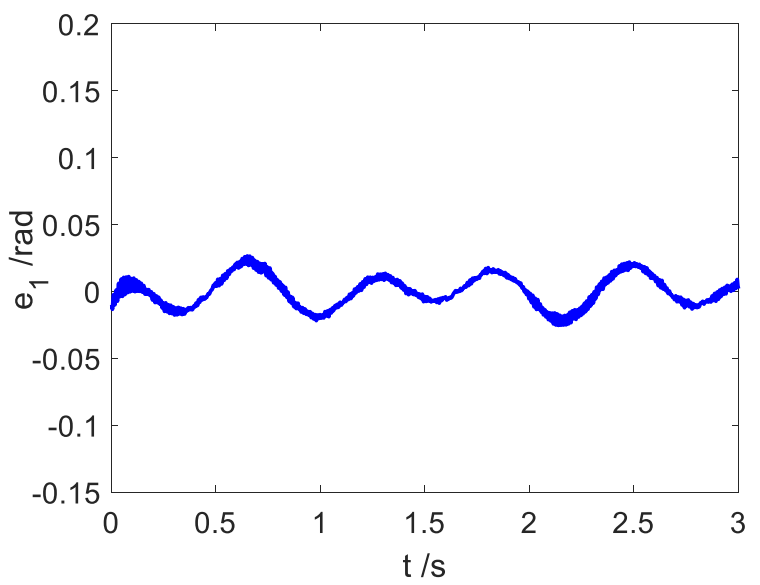

Figure 4. Error of tracking sine signal using RBFNNB.

\section{Conclusion}

For a class of nonlinear mismatched systems, we proposed a new RBF NN-based backstepping adaptive control. We designed the adaptive controller combining RBF NN and backstepping control, and analyzed its stability. In simulation analysis, sine signal is chosen as input signal, system has larger tracking error and worse adaptive tracking ability when using PID control, but system has smaller tracking error and better adaptive tracking ability when using RBFNNB, validating the superiority of the proposed control strategy. However, the 
system studied in this paper is the single input and single output (SISO) system, there is also multiple input and multiple output (MIMO) system in practical engineering. In this paper, we do not consider MIMO system, and this will be the focus of our future work.

\section{Conflict of interest}

The authors confirm that this article content has no conflict of interest.

\section{Acknowledgements}

This work was supported by National Natural Science Foundation of China(No.61074023), Natural Science Research Key Project of Universities in Anhui Province of China (No.KJ2015A297), Nature Science Foundation in Anhui Province of China (No.1508085MF130), the Engineering Technology Research Center of Optoelectronic Appliance in Anhui Province of China, Sichuan Institute of Aerospace System Engineering in Sichuan Province of China.

\section{References}

1. J. Wei, Y. Zhang, H. Bao, An exploration on adaptive iterative learning control for a class of commensurate high-order uncertain nonlinear fractional order systems, IEEE CAA J. Autom. Sin., 5(2) (2018) 618-627.

2. T. Kaczorek, Positivity and linearization of a class of nonlinear continuous-time systems by state feedbacks, Int. J. Appl. Math. Comput. Sci., 25(4) (2015) 827-831.

3. W. Lv, F. Wang, Finite-time adaptive fuzzy tracking control for a class of nonlinear systems with unknown hysteresis, Int. J. Fuzzy Syst., 20(3) (2018) $782-790$
4. J. Zhang, M. Lyu, T. Shen, Sliding mode control for a class of nonlinear multi-agent system with time delay and uncertainties, IEEE Trans Ind Electron, 65(1) (2018) 865-875.

5. K. Zhu, N.M. Qi, C.M. Qin, Adaptive sliding mode controller design for BTT missile based on backstepping control, Yuhang Xuebao, 31(3) (2010) 769-773.

6. J. Park, I.W. Sandberg, Universal approximation using radial-basis-function networks, Neural Computation, 3(2) (1991): 246-257.

7. S. Zhang, Z. Wu, An adaptive block backstepping control strategy for motorized spindles based on improved RBF neural network, Boletin Tecnico, 55(9) (2017) 432-439.

8. J. Liu, Radial basis function (RBF) neural network control for mechanical systems: Design, analysis and matlab simulation[M], Germany: Springer-Verlag Berlin Heidelberg, 2013.

9. W. Si, X. Dong, F. Yang, Decentralized adaptive neural control for high-order stochastic nonlinear strongly interconnected systems with unknown system dynamics, Inf Sci, 424 (2018) 137-158.

10. Y. Sun, B. Chen, C. Lin, Adaptive neural control for a class of stochastic nonlinear systems by backstepping approach, Inf Sci , 369 (2016) 748-764.

11. D. Mu, G. Wang, Y. Fan, Design of robust adaptive course controller for unmanned surface vehicle with input saturation, Int. J. Innov. Comput. Inf. Control, 13(5) (2017) 1751-1758.

12. X.J. Ren, R.C. Liu, Y. Chen, Z.Y. Yang, Robust adaptive control for a class of nonlinear systems using backstepping based on RBF neural network, Journal of Naval Aeronautical and Astronautical University, 23(6) (2008) 645-648.

13. Y. Hu, Y. Jin, P. Cui, RBF NN-based backstepping control for strict feedback block nonlinear system and its application, Lect. Notes Comput. Sci., 3174 (2004) 129-137. 